This is a self-archived - parallel published version of this article in the publication archive of the University of Vaasa. It might differ from the original.

\title{
An Adaptive Denoising Algorithm for Online Condition Monitoring of High-Voltage Power Equipment
}

Author(s): Hussain, Amjad; Ahmed, Zeeshan; Shafiq, Muhammad; Zaher, Ashraf; Rashid, Zeeshan; Lehtonen, Matti

Title: $\quad$ An Adaptive Denoising Algorithm for Online Condition Monitoring of High-Voltage Power Equipment

Year: $\quad 2020$

Version: Accepted manuscript

Copyright (C)2020 Taylor \& Francis. This is an Accepted Manuscript of an article published by Taylor \& Francis in Electric Power Components and Systems on 21 Oct 2020, available online:

http://www.tandfonline.com/10.1080/15325008.2020.1825554.

\section{Please cite the original version:}

Hussain, A., Ahmed, Z., Shafiq, M., Zaher, A., Rashid, Zee. \& Lehtonen, M. (2020). An Adaptive Denoising Algorithm for Online Condition Monitoring of High-Voltage Power Equipment. Electric Power Components and Systems 48(9-10), 1036-1048. https://doi.org/10.1080/15325008.2020.1825554 


\title{
An Adaptive De-noising Algorithm for Online Condition Monitoring of High Voltage Power Equipment
}

\author{
Amjad Hussain ${ }^{1, *}$, Zeeshan Ahmed ${ }^{2}$, Muhammad Shafiq ${ }^{3}$, Ashraf Zaher ${ }^{1}$, Zeeshan
}

Rashid $^{4}$, Matti Lehtonen 5

${ }^{1}$ American University of Kuwait, School of Engineering \& Applied Sciences, Safat, Kuwait

${ }^{2}$ Mississippi State University, Department of Electrical \& Computer Engineering, USA

${ }^{3}$ University of Vaasa, Department of Electrical Engineering \& Energy Technology, Vaasa, Finland

${ }^{4}$ The Islamia University of Bahawalpur, Department of Electrical Engineering, Bahawalpur, Pakistan

${ }^{5}$ Aalto University, School of Electrical Engineering, Espoo, Finland

*ghussain@auk.edu.kw

\section{Abstract:}

Partial Discharge (PD) diagnostic is an effective tool for condition monitoring of the high voltage equipment that provides an updated status of the dielectric insulation of the components. Reliability of the diagnostics depends on the quality of the PD measurement techniques and the processing of the measured PD data. The online measured data suffer from various inaccuracies caused by external noise from various sources such as power electronic equipment, radio broadband signals and wireless communication, etc. Therefore, extraction of useful data from the on-site measurements is still a challenge. This article presents a discrete wavelet transform (DWT) based adaptive de-noising algorithm and evaluates its performance. Various decisive steps in applying DWT based de-noising on any signal, including selection of mother wavelet, number of levels in multiresolution decomposition and criteria for reconstruction of the de-noised signals are taken by the proposed algorithm and vary from one signal to another without a human intervention. Hence, the proposed technique is adaptive. The proposed solution can enhance the accuracy of the PD diagnostic for HV power components.

Keywords: Online monitoring, Discrete Wavelet Transform (DWT), high voltage, insulation, signal de-noising 


\section{Introduction}

Modern life of today is highly dependent on electricity. Ranging from domestic (cooking, lighting, cooling, heating, etc.) to industrial applications (mobile communication, transportation, factories, irrigation, etc.), life cannot be imagined without an electric power. Therefore, it is a prime need of the day to have a reliable, efficient and continuous supply of electricity to the domestic and commercial loads. An electrical power network and its associated equipment are primarily made up of electrical conductors and insulations. The electrical components (power cables, transformers, switchgear and control gear, etc.) are exposed to various stresses such as thermal, electrical, ambient and mechanical stresses (also termed as TEAM stresses) during their operation. Such stresses slowly degrade the insulation elements that weaken their dielectric performance. The weakened part of the dielectric in the power components undergoes localized breakdowns that are called partial discharges (PD) and causes the emission of high frequency signals [1].

PD monitoring is considered as a reliable measure to anticipate the health of electrical insulation and hence the life expectancy of the electrical equipment [2]. Traditionally, offline techniques were used to measure the PD for electrical equipment, however such techniques have various drawbacks. For example, one drawback could be the inaccuracy could result in test data, due to high sensitivity of PD behaviour to the operating conditions. Moreover, new faults maybe introduced during a periodic maintenance cycle, which might lead to complete breakdown during this period [3]. Therefore, online condition monitoring techniques are required in order to track the continuously changing status of the insulation performance of critical power components. Such improved practices will help reducing the failure rate, unplanned outages, and consequently the economical burdens on the power utilities. 
Due to the current availability of modern micro- and nano-electronics, non-intrusive sensing technologies, effective digital signal processing and fast data communication techniques, new online condition monitoring concepts are being tested and deployed more easily, compared to 30 years ago. However, online measurement techniques suffer from a number of challenges. The most common challenge is the inclusion of different types of external noise, while performing online measurements. This is mainly due to using broadband non-intrusive sensors for conducting PD measurements. This causes the measured PD signals to contain a wide spectrum of frequencies that overlap with other ranges, e.g. those devoted to modern digital communication [4], [5]. When the measured data is swamped with significant amount of noise, this may result in erroneous interpretation and consequently, false diagnostics of the PD activity. In order to solve this problem, a sophisticated de-noising technique is needed so that useful PD information is extracted without the loss of accuracy [6]-[8].

Some of the de-noising techniques reported in [2], [9], and [10] have certain drawbacks. For example, they require human intervention to process the de-noising, and also they require large amounts of data to be extracted from PD tests that need to be stored for post processing. Each PD measurement has a significant data size (in MBs) when stored on computer memory. Dealing with 100 s of PD measurement require significant storage space and processing time. Moreover, transferring such a high volume of data from a remote location to the control centre is also challenging. Also, these techniques mainly result in loss of PD signal amplitude. Moreover, such techniques work for a particular noise type and cannot be generalized. A de-noising technique based on Wavelet Transform (WT) as reported in [5]-[7], [11], [12] provides a unique way of de-noising PD and other discharge signals such as arcing. WT is based on de-noising a signal while analysing 
it both in time and frequency domain, whereas well known Fast Fourier Transform (FFT) based denoising technique processes a signal in frequency domain only.

This paper is the continuation of the work done in [3], [7] and [8] as it presents an advanced algorithm for the de-noising of online measured electrical discharge signals based on Discrete Wavelet transform (DWT). Various challenges faced by other researchers in applying DWT based de-noising including selection of mother wavelet function, number of decomposition levels in multiresolution decomposition and criteria for reconstruction of the de-noised signals are addressed. The major contribution of the paper to propose an adaptive de-noising algorithm in Matlab which automates the determination of the above decisive parameters/ steps without a human intervention and which may vary from one signal to another. Hence, the proposed algorithm is adaptive by its nature. The performance of the enhanced technique is evaluated by testing it over a wide range of recorded PD signals.

The rest of the paper is organized as follow; Section 2 presents the experimental arrangement for measurement of the PDs that provides the measured data to be used for the de-noising. Section 3 describes the details of the DWT and the methodology of the proposed de-noising technique. Performance evaluation indices are highlighted in Section 4, which investigate the effectiveness of the de-noising technique. Applying the proposed technique to the measured PD signals is presented in Section 5. Results are presented in section 6 along with an in-depth analysis to discuss the performance indices and the validity of the proposed technique. The last section concludes the paper and highlights it's the most important advantages. 


\section{PD Measurement Setup}

The PD measurement setup was implemented in the HV laboratory and was also tested on the HV site of the Helsinki energy network, as illustrated in Fig. 1. The difference between the laboratory and the on-site test setups was the method used to measure the system voltage, a voltage divider was used in the former, while a capacitive probe was used in the latter. A variable supply of $0-12 \mathrm{kV}$ was used to apply the test voltage on the switchgear in the laboratory. The applied variable voltage enabled the change of voltage level so that the PD inception levels can be traced and acquired in suitable amplitude. Two sensors, i.e. high frequency current transformer (HFCT) and the $D$-dot sensor were used to record the PD signals and other high frequency transients, including noise. The $D$-dot sensor was installed within the bus bar compartment of the switchgear panel, while the HFCT was installed around the grounding terminals to measure the PDs. The operating principle, along with the electrical models and the transfer functions of the two sensors are given in [8], as well as in appendix A. Different types of PD faults including corona, voids in glass insulator, and surface discharge were created in the laboratory inside a medium voltage switchgear as reported in [13] and [8]. The PD signals swamped by various kind of noise, generated from the $10 \mathrm{kV}$ onsite switchgear and its connecting cables, were measured. For all the measurements, a high performance digital oscilloscope with sample rate of $2 \mathrm{GS} / \mathrm{s}$ was used for recording the signals. 


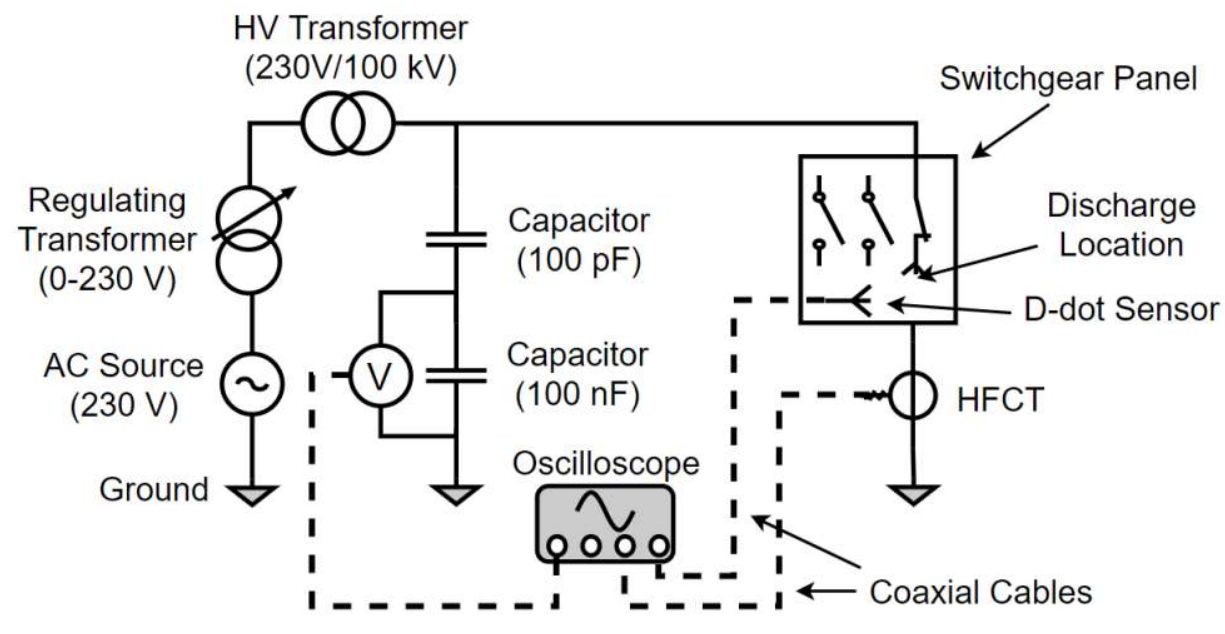

Fig. 1. PD Measurement setup

\section{Discrete Wavelet Transform (DWT)}

A wavelet is a wave with an effectively limited duration and zero mean value, as illustrated in Fig.

2. Unlike the sinusoidal waveforms, it is asymmetric. The discrete wavelet transform (DWT) converts a given signal into a set of scaled and shifted versions of a reference wavelet. The reference wavelet is termed as the mother wavelet and is mathematically written as [11],

$\psi_{a, b}(t)=\frac{1}{\sqrt{a}} \psi\left(\frac{t-b}{a}\right)$

In equation (1) $a$ is the amplitude scaling coefficient, whereas $b$ provides a shift in the mother wavelet $\psi(t)$. 

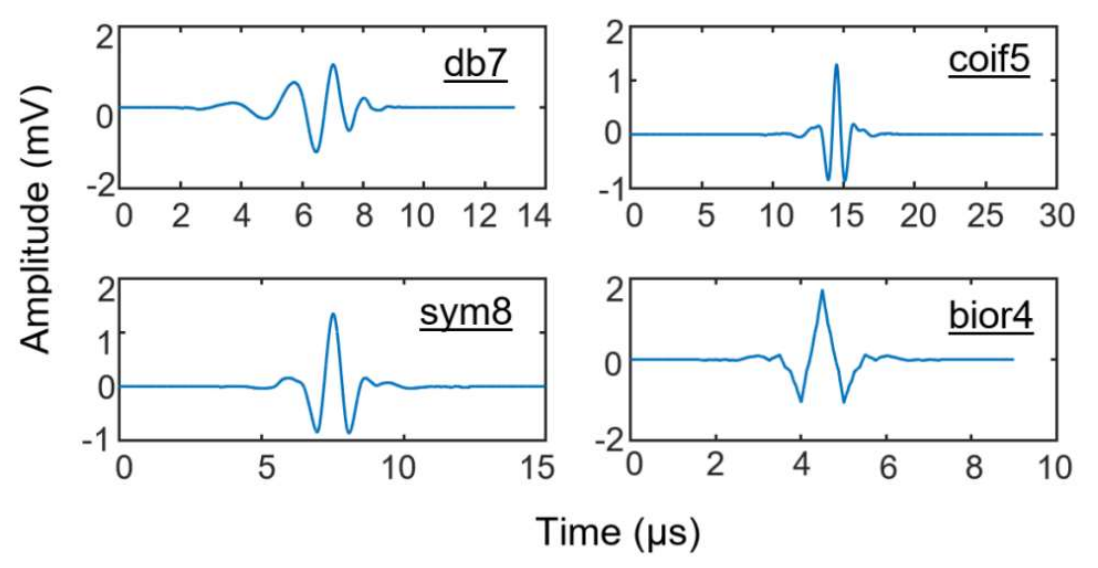

Fig. 2. Various wavelet functions

\subsection{DWT based de-noising}

Figure 3 shows the procedure for decomposition and reconstruction of a signal based on DWT, known as multiresolution signal decomposition (MSD). Considering a signal $\mathrm{X}[\mathrm{n}]$ to be de-noised; at first the signal is decomposed into high frequency $\left(\mathrm{H}_{0}\right)$ and low frequency $\left(\mathrm{G}_{0}\right)$ components through respective filters with down sampling at a certain level followed by further splitting of the low frequency component in a next level. The number of levels for the decomposition is defined by an algorithm, depending on the desired resolution or accuracy. The high frequency components are termed as detail or decomposition coefficients and the low frequency components are defined as approximations. In Fig. 3(a), X[n] is decomposed into two detail coefficients $\left(\mathrm{d}_{1}-\mathrm{d}_{2}\right)$, one at each level and an approximation $\left(\mathrm{a}_{2}\right)$ at the last level. Fig. 3(b) demonstrates the reconstruction procedure which is exactly opposite to the decomposition, where the detail coefficients and an approximation are added up after up sampling to reconstruct the de-noised signal [5]. 


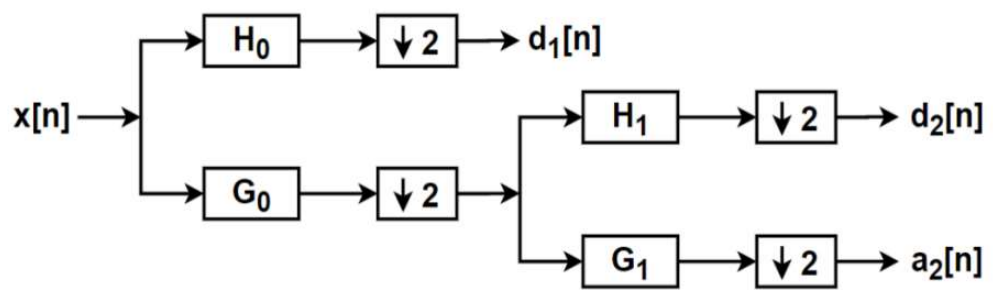

(a)

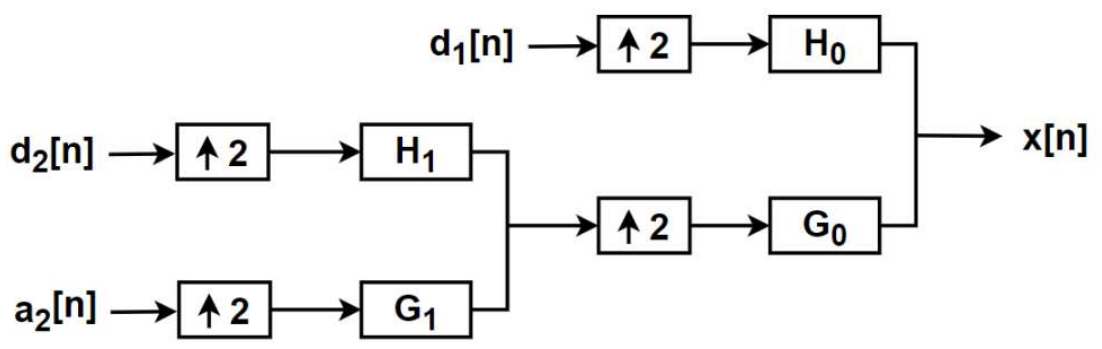

(b)

Fig. 3 (a) DWT based Multiresolution signal decomposition; (b) Reconstruction to obtain the denoised signal

Going through the above procedure, the following must be defined.

1. Number of levels: upto which the signal needs to be decomposed, which will further define the number of detail coefficients.

2. Selection of mother wavelet function: which must be close to the useful PD signal. It is possible to either use one mother wavelet for all the levels (known as multilevel) or unique mother wavelet for individual levels (known as level dependent).

3. Thresholding: to further refine the detail coefficients, to isolate the low amplitude noise from them.

Further description is presented in the following section. 


\subsection{Defining the Number of Decomposition Levels}

In most of manual de-noising techniques, based on DWT, the number of levels is selected by the user based on hit and trial. In an adaptive techniques, this has to be automated, i.e. the de-noising algorithm should define the number of decomposition levels itself. Therefore, a new method is proposed, based on the determination of a lowest frequency $\left(F_{\min }\right)$ below which the energy spectral density (ESD) is lower than 15\% of the peak ESD [6]. The adaptive algorithm uses this criteria to determine the number of decomposition levels.

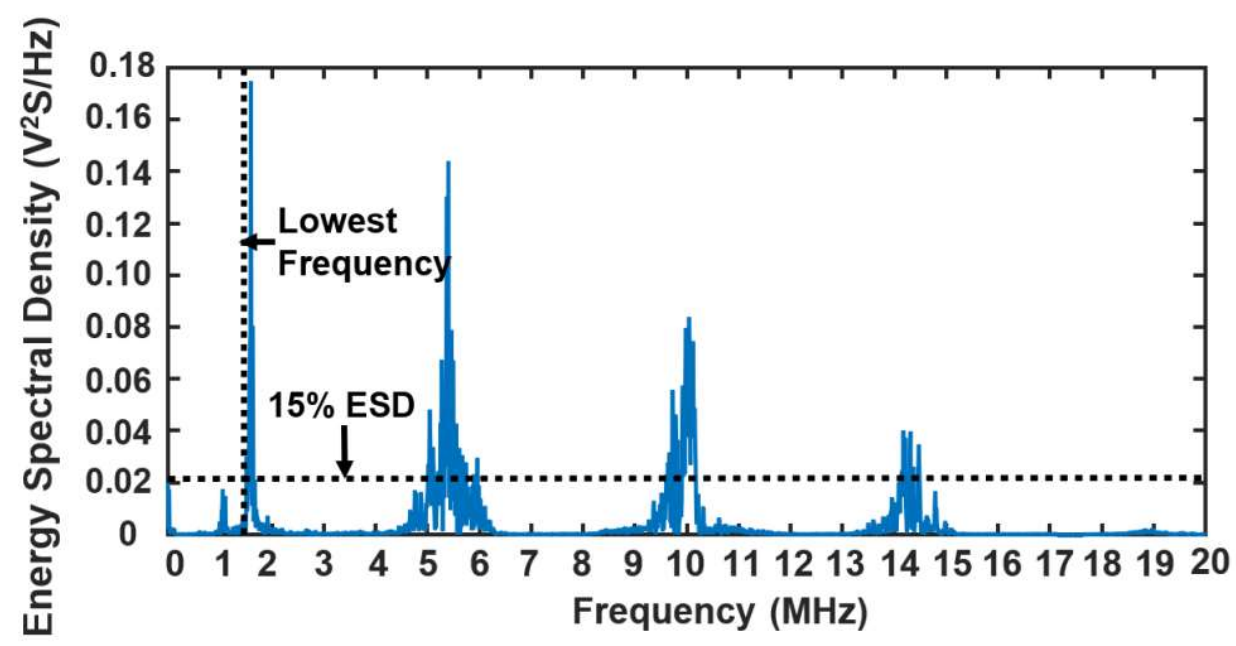

Fig. 4. Defining number of decomposition level using ESD

The following expression is used to calculate the number of decomposition coefficients,

$$
N=f i x\left[\log _{2}\left(\frac{F_{s}}{F_{\text {min }}}\right)\right]
$$

Where $F_{\min }$ is the minimum frequency below which ESD is less than $15 \%$ (in Fig. 4, it is marked as the vertical dotted line at $1.5 \mathrm{MHz}$ ) and $F_{s}$ is the sampling frequency of the signal.

\subsection{Selection of Mother Wavelet}

The mother wavelet is selected based on the similarity in wave shape of the PD signal to be denoised with the wavelet of a particular wavelet family, i.e. similarity in asymmetry, orthogonality, 
and compactness. It is tested and reported in the literature that Daubechies (db2-db15), Symlet (sym2-sym12) and Coiflets (coif1-coif15) possess properties which are the best suitable for denoising of high frequency PD and arcing signals [14], [5]. Therefore, these mother wavelets are preferred in this study. Further selection of wavelet from these families is based on the following two methods [6]:

- Correlation Based Wavelet Selection (CBWS)

- Energy Based Wavelet Selection (EBWS)

\section{Correlation Based Wavelet Selection (CBWS)}

As mentioned earlier, the similarity in wave shape between the PD signal and the wavelet function provides a basis for the selection of mother wavelet. Therefore, it is very logical to have such an algorithm which selects the mother wavelet based on this criteria. CBWS is based on maximizing the correlation coefficient between a PD signal, to be de-noised, and a wavelet function from a particular wavelet family and hence it can be an effective method [6]. The equation for calculation of correlation coefficient is given in the next section, equation (8). This technique can be used as a multilevel or level dependent. The CBWS was evaluated on 200 measured signals by comparing with three wavelet families, i.e. Daubechies (db2-db15), Symlet (sym2-sym12) and Coiflets (coif1coif15). Figure 5 illustrates the calculated correlation coefficients for 200 PD signals with respect to these wavelet functions. It can be observed that the lower order wavelet functions produced higher correlation coefficients, i.e. Daubechies- db2, db3; Symlets- sym2, sym3; Coiflets- coif1. 


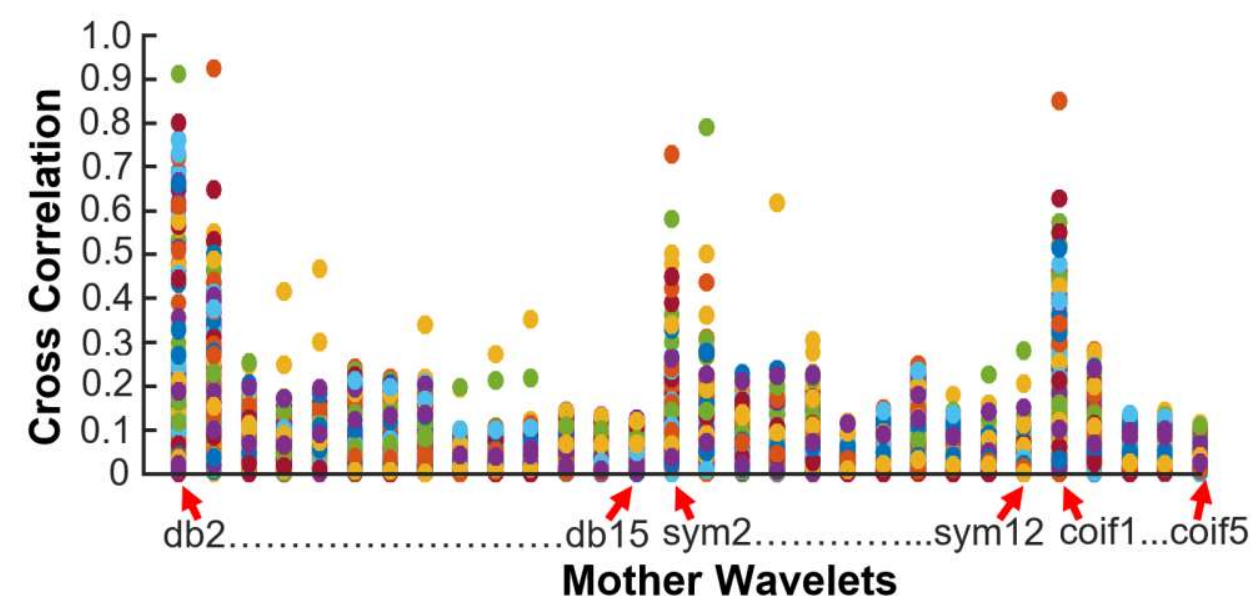

Fig. 5 Correlation Coefficient for different wavelet functions

The type of a sensor used to measure PD signals determines the wave shape and hence a particular mother wavelet function can be associated with a particular sensor [15]. A PD signal measured by an HFCT sensor has damped type oscillating waveform. Due to $R L C$ (equivalent resistance, inductance, and capacitance of the sensor) circuit behavior of HFCT and $D$-dot, their response to PD signals exhibit an attenuated oscillation, with a decaying rate that depends on the values of R, L, and $\mathrm{C}$, where $\mathrm{R}$ acts a damping factor for the sustained oscillation, introduced by the LC combination. Generally, an external damping is provided to remove the oscillations in a calibrated environment. The $R L C$ equivalent model of the HFCT is shown in Appendix I (Fig. A1) where the transfer function of the HFCT is presented to highlight its operation. Similarly, the transfer function of the $D$-dot sensor along with its equivalent circuits are presented in Appendix I (Fig. A2). Figures 6 shows the comparison between a measured PD signal with $\mathrm{db} 3$ wavelet. It can be observed that both the wave shapes are consistent, which also confirms that lower order wavelet functions have similar wave shapes to PD signals. 


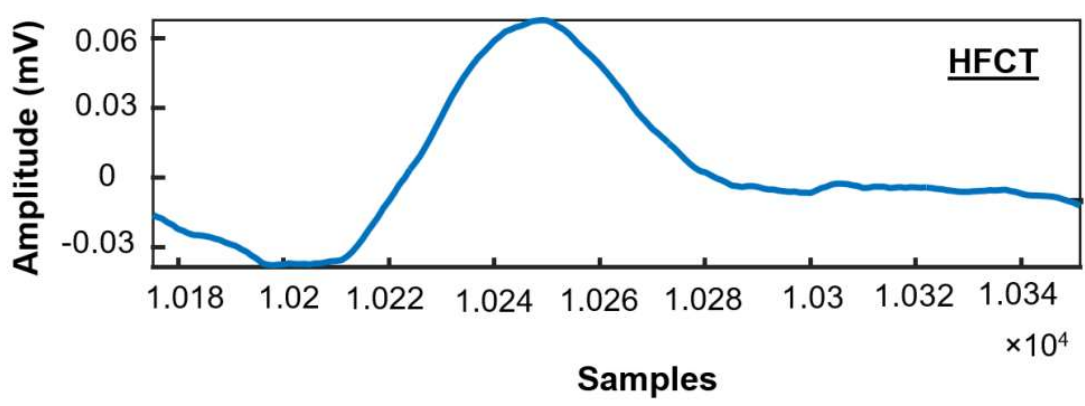

Fig. 6(a). PD pulse measured by HFCT sensor

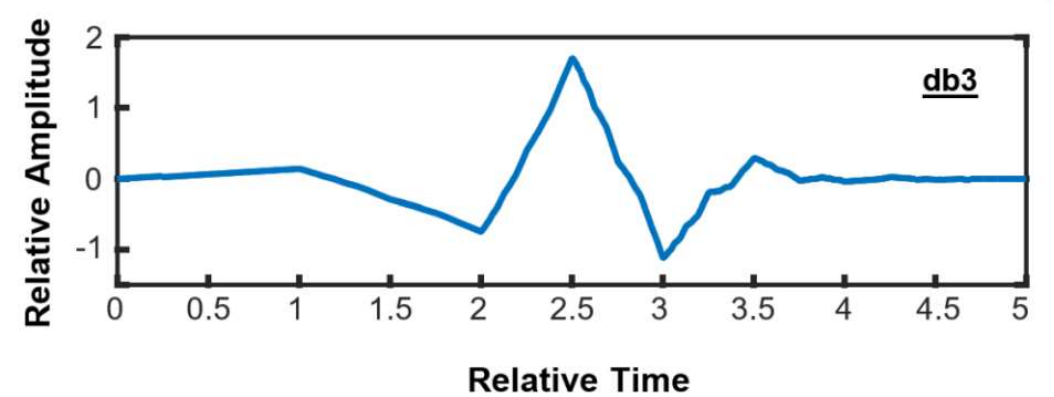

Fig. 6(b). Wavelet function 'db3'

\section{Energy Based Wavelet Selection (EBWS)}

The EBWS is mainly a level dependent wavelet selection method in which relative percentage energy content of the approximation is calculated, followed by selecting the wavelet that gives its maximum value [6], [16]. The energy contents can be calculated from the following relation, $E_{a}=\frac{\sum_{k} a_{j, k}^{2}}{\sum_{k} a_{j, k}^{2}+\sum_{i=1}^{j} \sum_{k} d_{j, k}^{2}}$

Where $a_{j}=\left[a_{j, 1}, a_{j, 2}, \ldots \ldots \ldots a_{j, k}\right]$ and $d_{j}=\left[d_{j, 1}, d_{j, 2}, \ldots \ldots \ldots d_{j, k}\right]$ are amplitudes of approximations and details coefficients, respectively at level $j$ and $k$ represents sample number. 


\subsection{Thresholding of Detail Coefficients}

Threshold in DWT based de-noising is the minimum amplitude for a certain detail coefficient under which the values are suppressed or replaced with zero. The small values in detail coefficients are mainly white noise. Sometimes, improper thresholding may lead to loss of information. Automated thresholding techniques presented in [17], [18] describe the way threshold levels are determined. However, in this paper, thresholding is not used.

\section{Performance Evaluation Indices}

To evaluate the effectiveness and performance of a de-noising technique, the following performance evaluation indices are calculated.

\subsection{Signal to Noise Ratio (SNR)}

The SNR is calculated as in equation (4) [5]. In this relation $X_{d e}$ is the de-noised signal, $X$ is the original measured signal, and $\mathrm{N}$ is the total number of samples. A higher values of SNR indicates better or improved de-noising.

$$
S N R=10 \times \log \left[\frac{\sum_{i=1}^{N} X_{d e}^{2}(i)}{\sum_{i=1}^{N}\left[X(i)-X_{d e}(i)\right]^{2}}\right]
$$

\subsection{Reduction in Noise Level}

The reduction in the noise level in a measured PD signal can be found using the following equation, that determines the extent of noise suppressed [5].

$$
d B s=10 \times \log \sum_{i=1}^{N} \frac{1}{N}\left[X(i)-X_{d e}(i)\right]^{2}
$$


The decibel $(\mathrm{dB})$ is a unit of measurement used to express the ratio of one value of a property to another on a logarithmic scale for measurements such as voltage, power, sound etc. It can also be used to express a change in a value such as in equation (5).

\subsection{Mean Square Error (MSE)}

The mean square error is determined as follows.

$$
M S E=\frac{\sum_{i=1}^{N}\left[X_{d e}(i)-X(i)\right]^{2}}{N \times P_{n o i s e}}
$$

Where $P_{\text {noise }}$ is the average power of the noise. If the MSE is unity, it means the noise is removed effectively.

\subsection{Reduction in PD amplitude}

The reduction in PD signal amplitude levels after the de-noising are calculated as,

$\%(-A m p)=\frac{X-X_{d e}}{X} \times 100$

\subsection{Correlation Coefficient (CC)}

As the name implies, the $\mathrm{CC}$ determines the degree of similarity between the two signals being compared. While evaluating the performance of the de-noising technique, the original measured signal is compared to the de-noised signal and a CC using the following relation, is calculated [5], [6]

$$
\gamma=\frac{\sum_{i=1}^{N}\left(X_{i}-\bar{X}\right)\left(X_{d e_{i}}-\bar{X}_{d e}\right)}{\sqrt{\sum_{i=1}^{N}\left(X_{i}-\bar{X}\right)^{2} \times \sum_{i=1}^{N}\left(X_{d e_{i}}-\bar{X}_{d e}\right)^{2}}}
$$

where $\gamma$ is $\mathrm{CC}$ and $\bar{X}$ and $\bar{X}_{d e}$ represent the mean values of original and de-noised signals respectively. 


\section{Implementation of the proposed Adaptive De-noising Algorithm}

Figure 7 outlines the algorithm for the proposed self-adaptive de-noising which is implemented to de-noise PD signals measured in the laboratory as well as on-site. The same algorithm can be applied to any online measured data including arcing, corona, internal partial discharges due to voids, and cracks or loose connections. The de-noising procedure discussed in the Section 3 is implemented using an adaptive approach for the selection of mother wavelet, deciding on the number of decomposition levels, and selection of detail coefficients through the de-noising algorithm. The whole de-noising process is automated as follows:

- To remove the discrete spectral interference (DSI) noise introduced by the radio, TV or telecommunication broadband signals, band stop filters tuned at their frequencies, are applied.

- The noise introduced by power electronic equipment are periodic pulses that are repetitive at a constant time interval. This noise can be eliminated by using gating technique. [19]

- The output signal obtained at the gating, termed as refined data is mainly comprised of discharge transient signal with added white noise and random pulses. Then FFT analysis is applied on the refined signals to get information about the dominant frequency contents in the signal to be de-noised.

- In the next step, the number of decomposition levels is determined based on the criteria given in section 3.2 .

- A mother wavelet is chosen based on the criteria given in section 3.3. It is also concluded in Section 6.2 that selecting/ defining one mother wavelet for all the decomposition level is an effective approach.

- Decompose the signal using standard DWT code as represented in section 3.1. 
- Next step is the most important task in this algorithm, i.e. inspection and selection of detail coefficients for reconstruction of de-noised signal. This is done by comparing the frequency content and amplitude of each detail coefficient with the original signal. For the comparison, certain frequency and amplitude minimum thresholds are defined (for example 20\%) of the original signal's frequency and amplitude. Any detail coefficient which has frequency and amplitudes higher than this threshold, they will be selected for reconstruction.

- In the final step, the de-noised signal is reconstructed by adding up all the selected detail coefficient, as shown in Fig. 3(b).

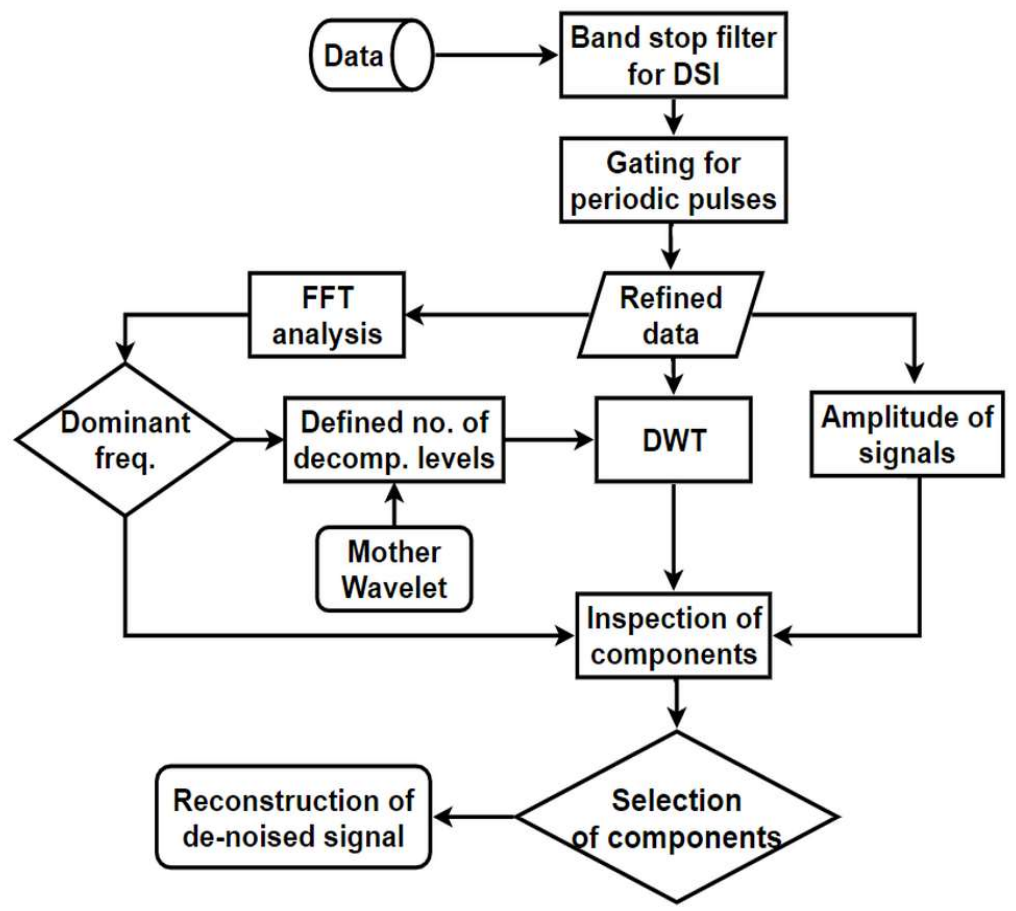

Fig. 7. Flow chart of self adaptive de-noising in online condition monitoring

Figure 8 illustrate the effect of changing the frequency and amplitude thresholds of the measured signals on MSE, CC, and NSR. It is recommended to use $20 \%$ and $60 \%$ thresholding for the amplitude and the frequency, respectively due to the stability of above parameters below these thresholds. 


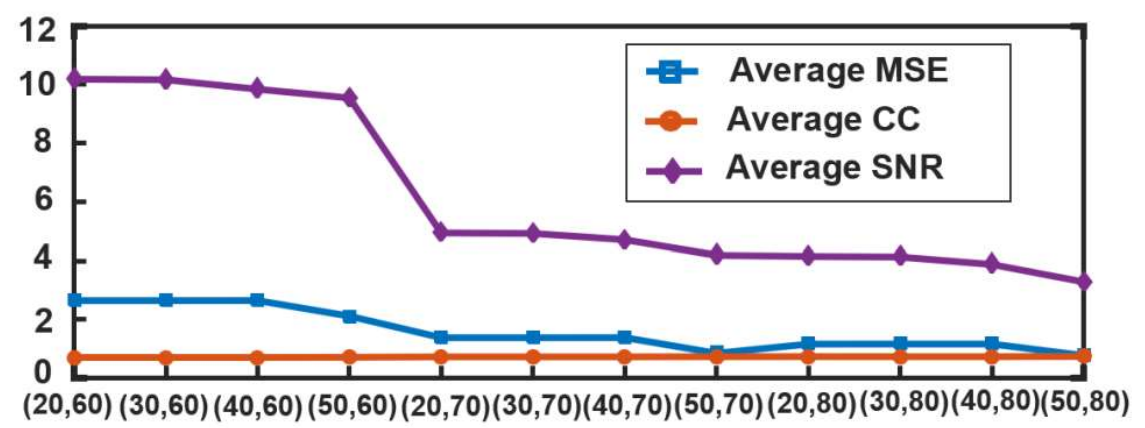

(Frequency, Amplitude) Limits

Fig. 8. Performance indices against various frequency and amplitude limits

\section{Results and Discussion}

\subsection{PD signal and Simulated Noise}

Figure 9 (a) shows a typical PD signal measured in the laboratory. This signal has very low content of external noise due to shielding of the HV laboratory facility. A typical white noise is simulated and added up to the PD signal, as shown in Fig. 9(b) and (c) respectively. The resulting signal shown in Fig. 9(c), named as S1, is similar to PD signals measured on site. A total of 200 PD signals, measured on site, were evaluated and performance indices were calculated to study the performance of the proposed adaptive de-noising technique.

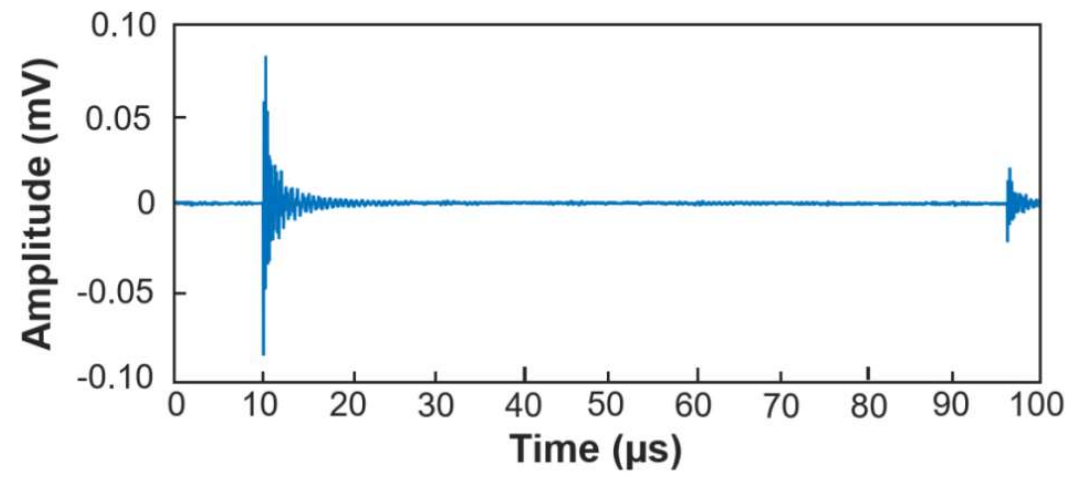

(a) 


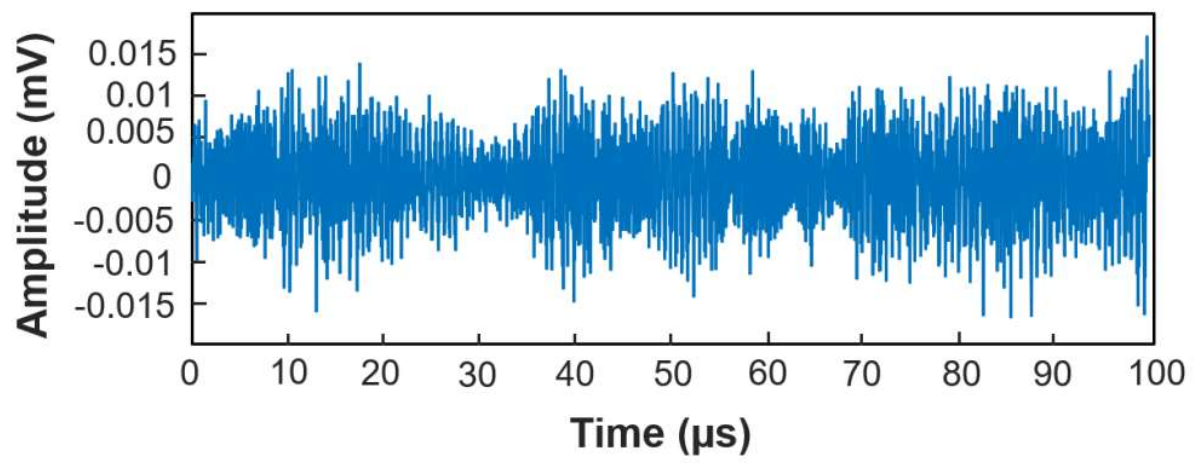

(b)

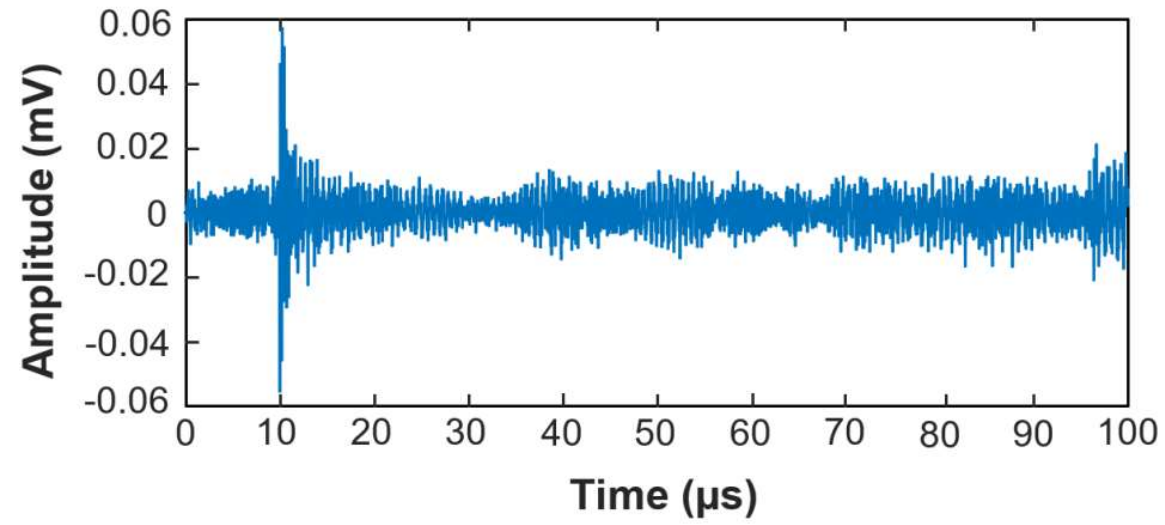

(c)

Fig. 9. PD Signal to be analysed (a) the PD signal measured in laboratory (without noise), (b) Simulated white noise, (c) PD signal mixed with noise

\subsection{Selection of Mother Wavelet based on EBWS vs. CBWS}

The PD signal S1, shown in Fig. 9 (c), is de-noised using two manual de-noising approaches by selecting the mother wavelet based on EBWS and CBWS. The number of levels was selected using the method described in section 3.2, which resulted in 9 levels. EBWS analysis lead to choosing different mother wavelets at each decomposition level (sym8, db11, db9, db2, db2, db3, sym5, db2, and sym2 respectively), whereas ' $\mathrm{db} 2$ ' was selected based on CBWS multilevel de-noising approach. Both de-noising approaches de-noised the signal S1 effectively as shown in Fig. 10 and 11; however, after a closer observation at both de--noised signals, it can be seen that the de-noised signal through EBWS has a better wave shape correlation with the original PD signal compared to the de-noised signal through CBWS. Therefore, the performance indices calculated from the two de- 
noised signals differ slightly as given in Table 1 . However, it can be seen that the difference between the performances of the two techniques is not so significant but the computation complexity and time taken by the processor is significantly higher for EBWS compared to CBWS. Therefore, it is proposed that the CBWS can be used without compromising the de-noising quality significantly. Further, it is also seen that a pre-defined mother wavelet associated with the measuring sensor and the nature of fault, such as PD, will further reduce the computation time. In order to verify this assumption, 200 signals were de-noised by the following two methods:

a. De-noising based on mother wavelet selected for each signal determined by CBWS

b. One pre-defined mother wavelet for all 200 signals (either $\mathrm{db} 2$ or $\mathrm{db} 3$ ).

The average values for performance indices calculated for 200 signals are given in Table 2. It can be observed that there is no significant difference between them. Hence, using a more time consuming technique such as CBWS is not recommended. Therefore, it is concluded that a preselected mother wavelet such as $\mathrm{db} 2$ or $\mathrm{db} 3$ produces effective results for de-noising.

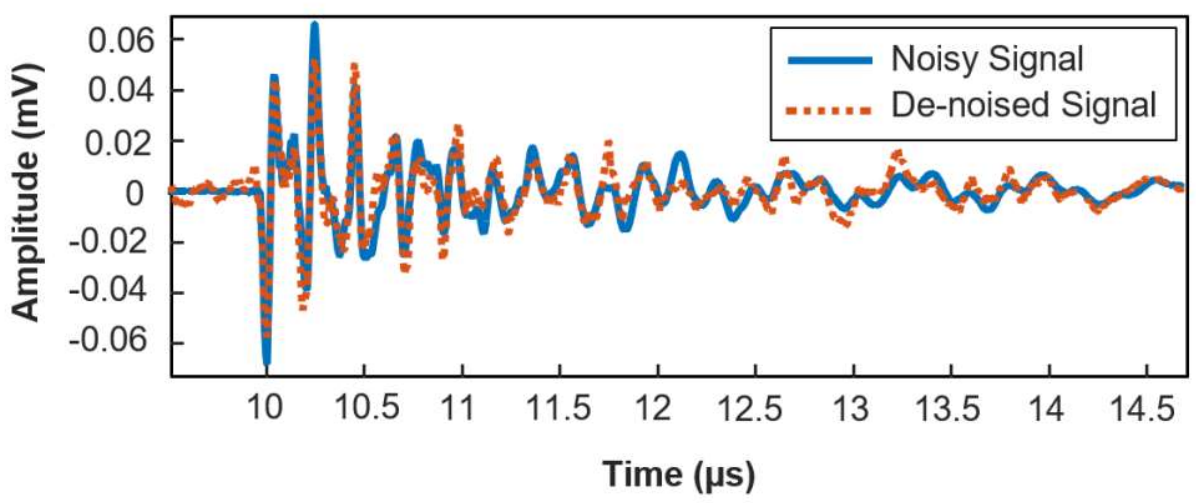

Fig. 10. De-noising based on EBWS 


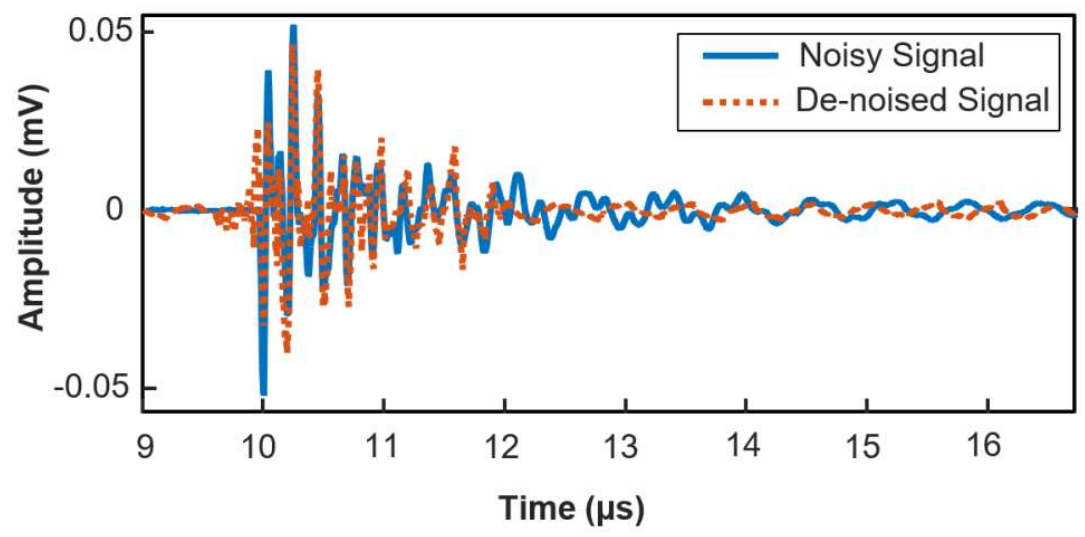

Fig. 11. De-noising based on CBWS

Table 1. Performance indices for EBWS and CBWS based de-noising

\begin{tabular}{cccccc}
\hline & CC & MSE & Reduc. in Amp. (\%) & Reduc. in noise (dB) & SNR \\
& & & & & \\
\hline EBWS & 0.689 & 0.261 & 11.219 & -52.898 & 2.629 \\
\hline CBWS & 0.678 & 0.233 & 13.137 & -53.313 & 2.13 \\
\hline
\end{tabular}

Table 2. Performance indices for evaluation of CBWS and pre-defined wavelet selection

\begin{tabular}{cccccc}
\hline De-noising & CC & MSE & Reduc. in Ampl. & Reduc. in Noise (dB) & SNR \\
\hline db2 & 0.7279 & 0.4010 & 5.8059 & -56.2410 & 5.2109 \\
\hline CBWS & 0.7286 & 0.3978 & 7.9921 & -56.2589 & 5.2161 \\
\hline $\mathbf{d b 3}$ & 0.7254 & 0.4025 & 9.4831 & -56.2189 & 5.1831 \\
\hline
\end{tabular}

\subsection{Evaluation of the Proposed Adaptive De-noising Technique}

Considering the PD signal shown in Fig. 9(c) which includes a simulated noise, the proposed denoising algorithm is evaluated. The peak to peak amplitudes of the signal are given in the same figure. In order to evaluate the frequency contents in the given signal, FFT analysis is performed as shown in Fig. 12. The dominant frequency for this signal, at which the harmonic content is 
maximum, is $1.5 \mathrm{MHz}$. The mother wavelet of the signal is pre-defined as ' $\mathrm{db} 2$ ' based on the arguement in the previous section. The number of levels for decomposition is determined through the methodology presented in section 3.2 and found to be 15 . Therefore, when DWT is applied, the signal is decomposed into 15 detail coefficients and an approximation, as shown in Fig. 13. For reconstruction of the de-noised signal, frequency comparison determined that details coefficients d7-d9 have harmonics higher than the pre-defined threshold limits, whereas the amplitude comparison determines that detail coefficient $\mathrm{d} 5-\mathrm{d} 7$ have amplitudes higher than the amplitude threshold limits. Thus d5-d9 are selected for the reconstruction of the de-noised signal. This shortlisting based on frequency and amplitude comparison results in reducing computation time as well as eliminating/ removing all the external noises. It can be clearly observed that the algorithm de-noised the signal S1 successfully and recovered the PD pulse buried in noise. The de-noised signal is plotted along with the noisy PD signal for comparison in Fig. 14 (a-d).

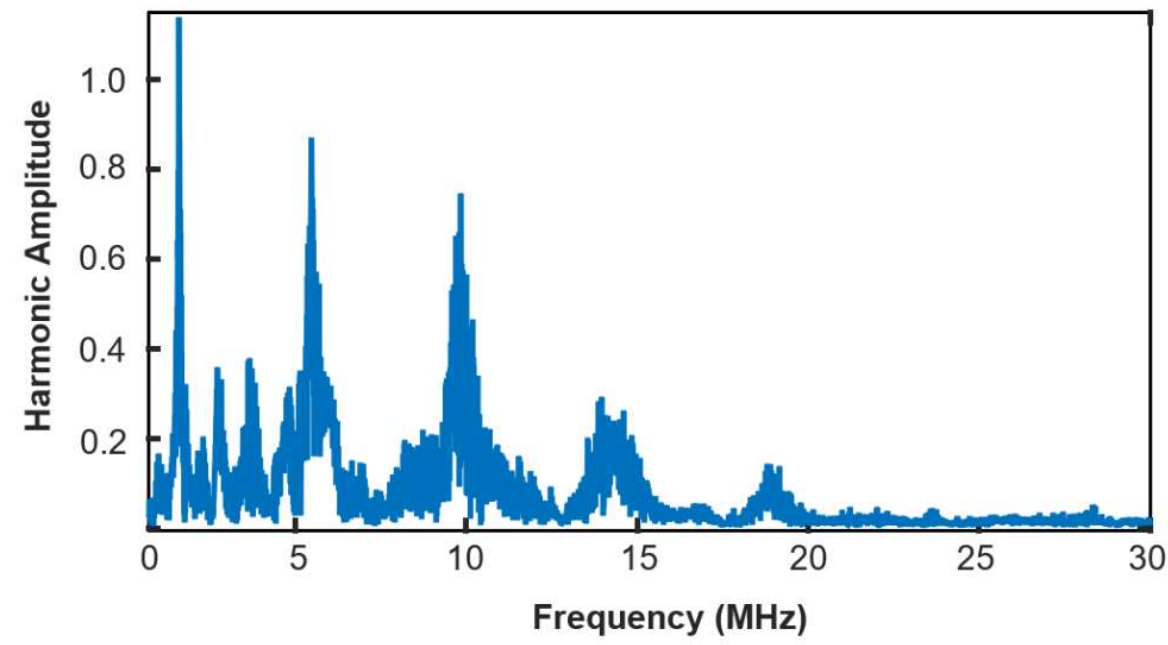

Fig. 12. FFT of signal S1

Figure 14(a) shows the actual measured PD signal, Fig. 14(b) is the PD signal after adding the simulated noise, and in Fig. 14(c) the de-noised signal can be seen, which shows that the denoising has also removed high frequency low level noise from the original PD signal which usually contain 
no information about a PD fault. The information about a PD activity and PD energy is reflected in the first few pulses generally. Therefore, the de-noising is very effective. Also Fig. 14(d) proves that the pulse shape of the measured PD signals is retained. The performance indices are calculated and given in Table 3. The calculated $\mathrm{CC}$ and reduction in amplitude are 0.821 and $1.5 \%$ illustrating excellent similarity between the de-noised signal and the original PD signal.

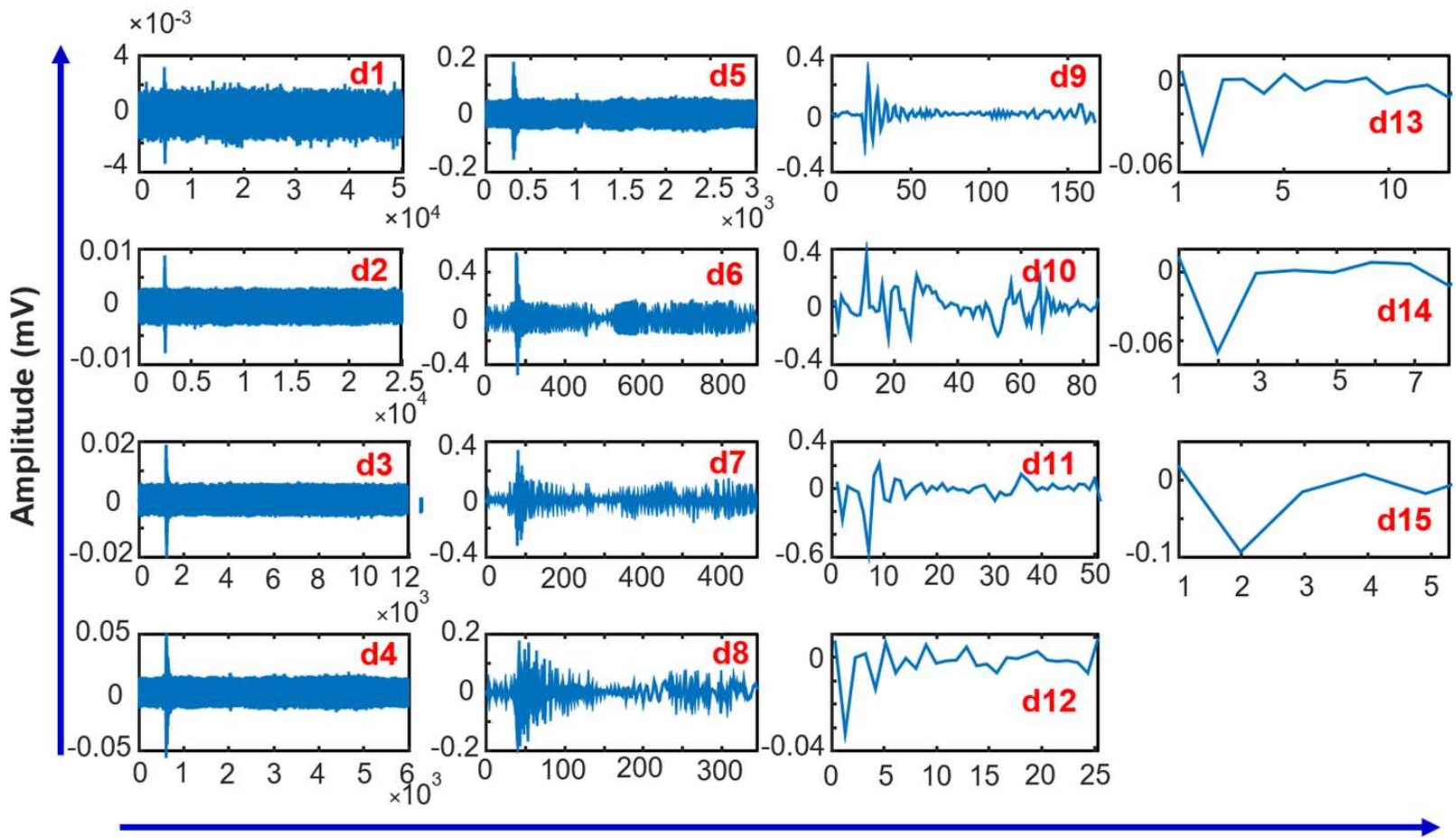

No. of Samples

Fig. 13 Decomposition of signal S1 using db2 and 15 levels

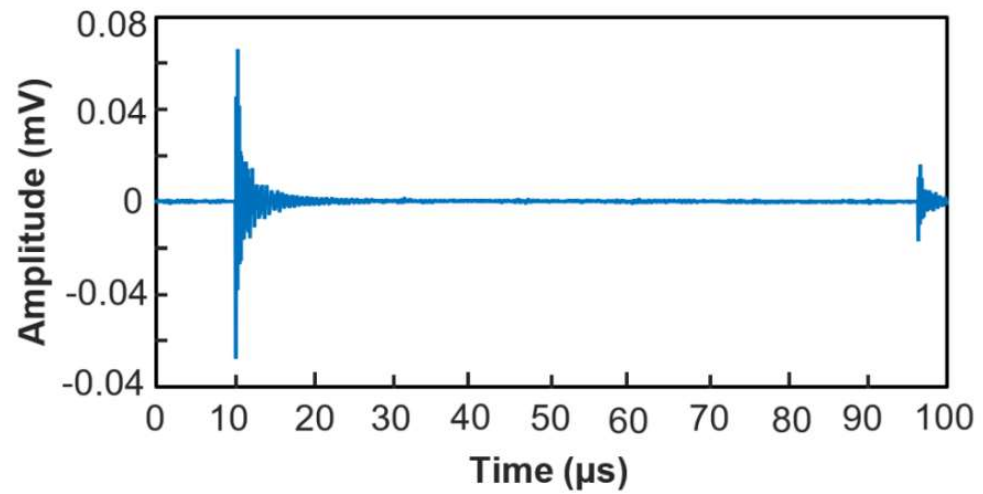

(a) 


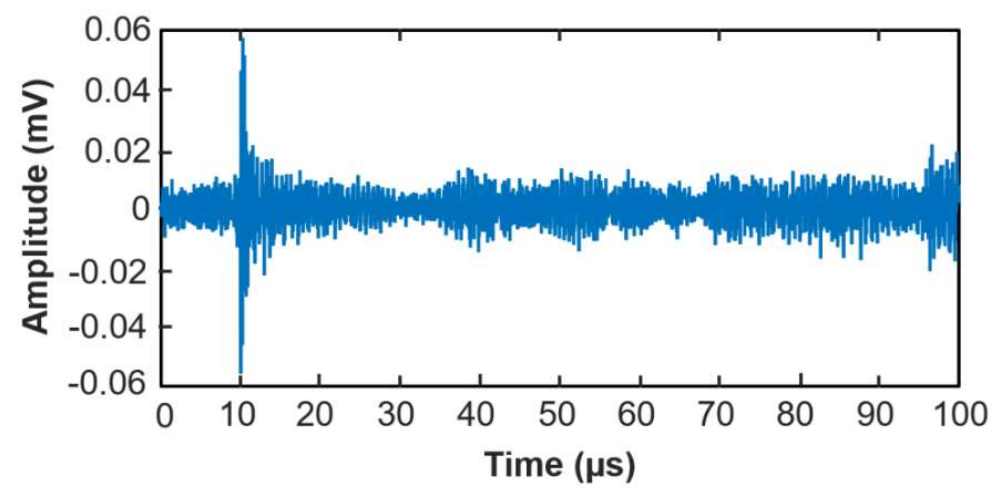

(b)

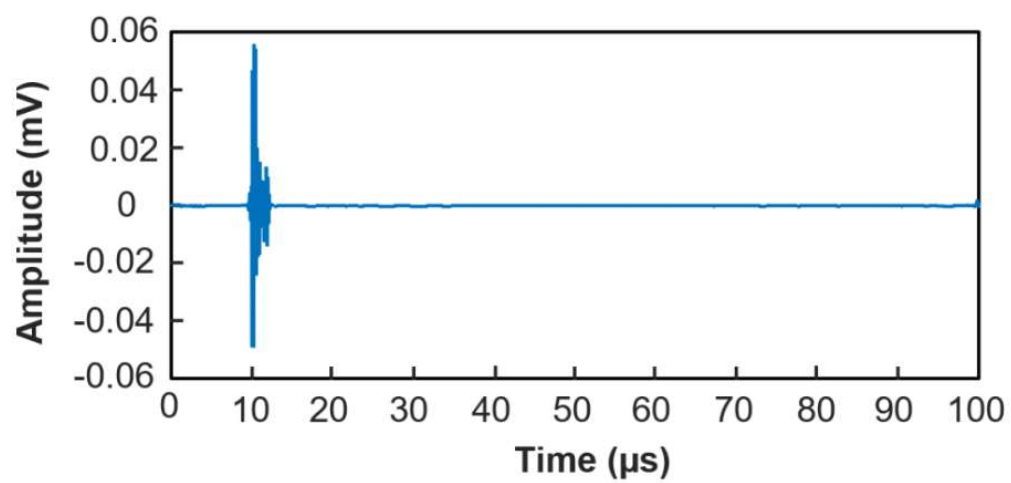

(c)

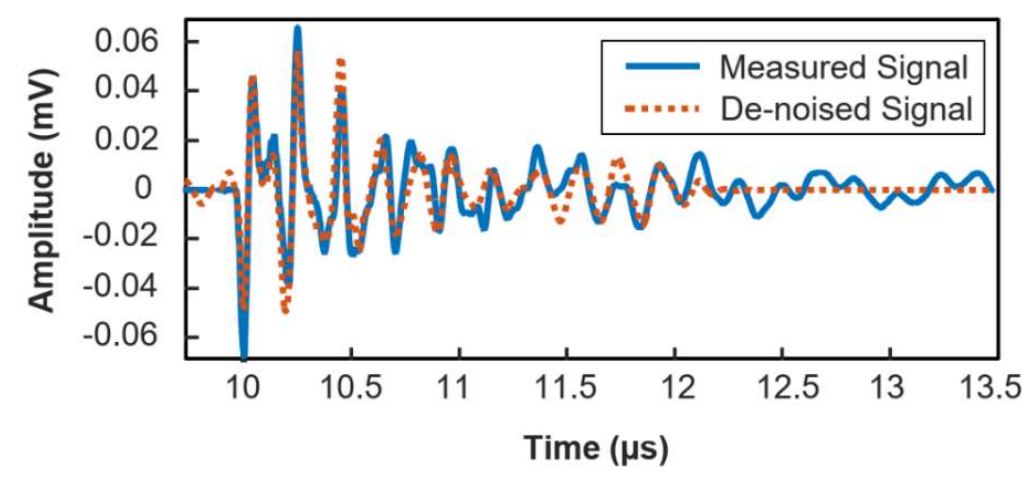

(d)

Fig. 14. (a) PD signal captured by the sensor, (b) the PD signal with added noise, (c) De-noised signal, (d) Comparison between the measured PD signal and the de-noised signal (after removing the added noise)

Table 3. Performance indices of the de-noised signal S1 through adaptive de-noising

\section{MSE Reduc. in Ampl. (\%) Reduc. in Noise (dB) SNR}

$\begin{array}{llllll}\text { S1 } & 0.821 & 0.120 & 1.521 & -56.31 & 3.29\end{array}$


The histograms given in Fig. 15 (a-e) show the performance indices calculated after de-noising the 200 signals through the proposed adaptive de-noising algorithm. The average values of the same are given in Table 4 . The average values are quite promising, proving the effectiveness of the de-noising algorithm.

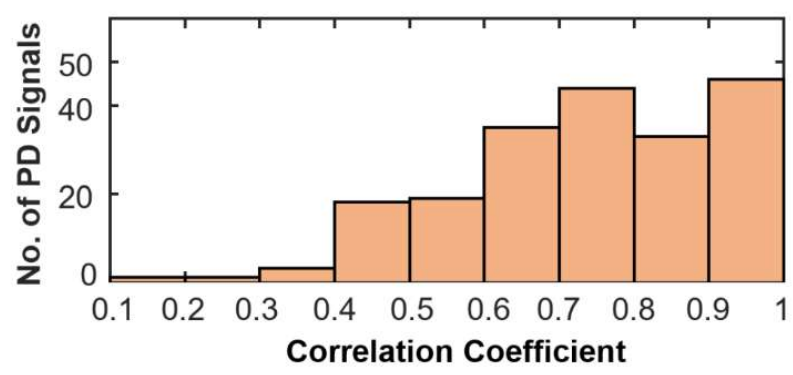

(a)

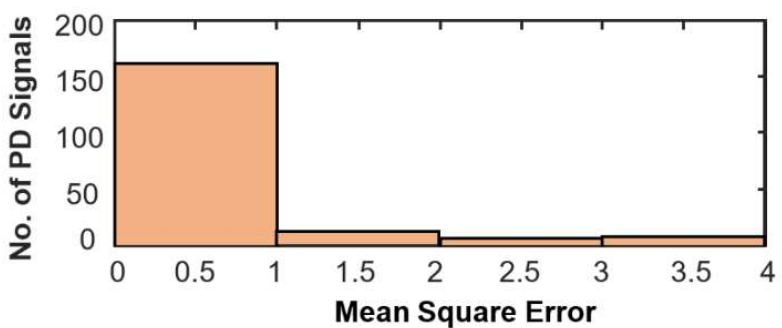

(b)

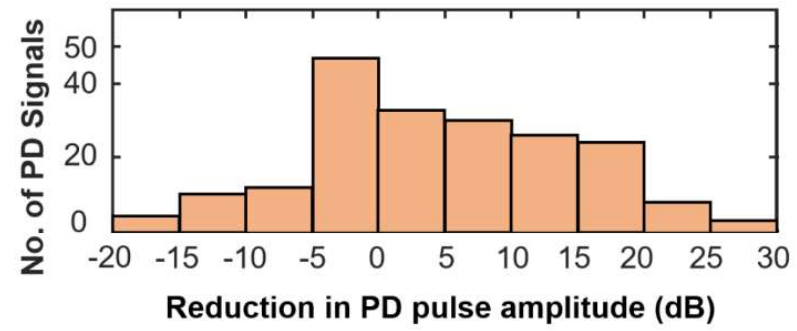

(c) 


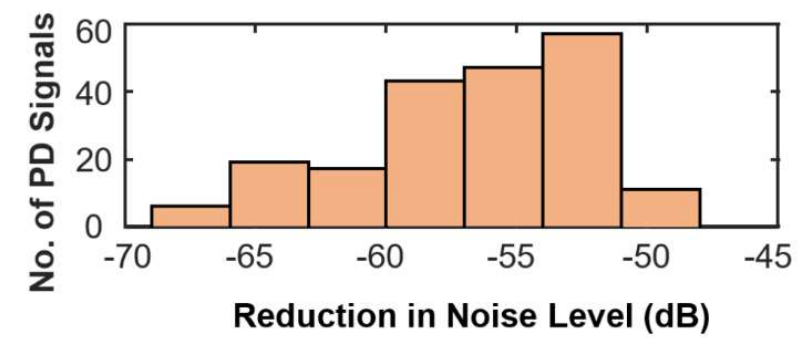

(d)

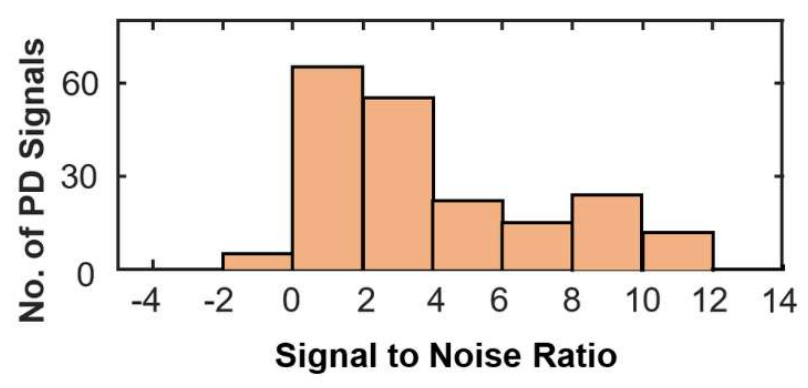

(e)

Fig. 15. Performance indices for 200 signals de-noised by the adaptive de-noising technique, (a) correlation coefficient, (b) Mean square error, (c) Reduction in PD pulse amplitude, (d) Reduction in noise level, (e) Signal to noise ratio

Table 4. Average values of performance indices of the 200 de-noised signals

\begin{tabular}{cccccc} 
& CC & MSE & Reduc. in Ampl. (dB) & Reduc. in Noise (dB) & SNR \\
\hline Avg. & 0.786 & 0.75 & 3.87 & -49.10 & 3.77 \\
\hline
\end{tabular}

\section{Conclusion}

This paper presents an adaptive algorithm for de-noising of the online measured signals to detect incipient faults such as PD and low power arcing. During online measurements, various types of external noises affect the measured signals and may compromise the accuracy of fault detection. 
Therefore, de-noising is of significant importance in online condition monitoring. The proposed algorithm automates most of the challenges tasks during the implementation of DWT based denoising, which include optimum selection of mother wavelet, deciding on the number of decomposition levels, and finding the best criteria to select detail coefficients for re-constructing denoised signals. These parameters vary from one signal to another and the algorithm determines them effectively without a human intervention, hence it is an adaptive method. The proposed algorithm is evaluated for a wide range of signals collected form laboratory as well as during on-site measurements. Various performance indices are calculated to validate the effectiveness of the proposed algorithm.

The two well-known techniques for the selection of mother wavelet are evaluated and it can be concluded that CBWS based mother wavelet selection produces satisfactory results while requires less computation time. Moreover, a specific mother wavelet determined from the CBWS can be adopted for all the PD signals measured by a dedicated sensor. The proposed de-noising technique is based on Matlab Wavelet toolbox, which can be translated and embedded in a specific on-site intelligent electronic device (IED), using proper software tools.

The developed de-nosing algorithm can be used in the real world application of online condition monitoring and diagnostics for power transformers, switchgear panels, power cables, as well as electrical motors and generators. The proposed HFCT and $D$-dot sensors can be used efficiently to measure the high frequency transient signals from on-site equipment. The recorded signals can then be processed and de-noised in a data acquisition unit before being sent to the control room via SCADA system. Unlike the fault protection system, the processing time is not critical in PD monitoring systems because such faults develop slowly and it make take hours, weeks or even months until they lead to complete breakdown of a dielectric. Therefore, readings taken per minute 
suffice the purpose of monitoring an insulation. The proposed de-noising algorithm takes only a few 10s of milliseconds to perform the de-noising on a microprocessor, which is quite suitable for the application. The cost of the proposed implementation can be optimized by using one acquisition and processing unit for multiple sensors and multiple equipment. The implementation of the proposed technique will significantly improve the detection of incipient faults in major power system equipment, which could help in reducing the number of unplanned outages due to sudden failure of electrical equipment.

\section{References:}

[1] Luo, Y.; Li, Z.; Wang, H., 2017. A Review of Online Partial Discharge Measurement of Large Generators. Energies, 10(11), art. No. 1694.

[2] Sriram, S.; Nitin, S.; Prabhu, K.M.M.; Bastiaans, M.J., 2015. Signal De-noising Techniques for Partial Discharge Measurements. IEEE Trans. Dielec. and Electr. Insul., 12(6), pp. 1182-1191.

[3] Hussain, G.A; Shafiq, M.; Kay, J.A.; Lehtonen, M., 2015. Preemptive Arc Fault Detection Techniques in Switchgear- Part III: From the Laboratory to Practical Installation. IEEE Trans. Ind. Appl., 5(3), pp. 2615-2623.

[4] Kumpulainen, L.; Hussain, G.A.; Lehtonen, M.; Kay, J.A., 2013. Preemptive Arc Fault Detection Techniques in Switchgear and Controlgear. IEEE Trans. Ind. Appl., 49(4), pp.1911-1919.

[5] Satish, L.; Nazneen, B., 2003. Wavelet-based De-noising of Partial Discharge Signals Buried in Excessive Noise and Interference. IEEE Trans. Dielec. and Electr. Insul., 10(2), pp. 354-367.

[6] Cunha, C.F.F.C.; Carvalho, A.T.; Petraglia, M.R.; Lima, A.C.S., 2015. A New Wavelet Selection Method for Partial Discharge De-noising. Electric Power Sys. Research, 125, pp. 184-195.

[7] Hussain, G.A.; Shafiq, M.; Kumpulainen, L.; Mahmood, F.; Lehtonen, M., 2015. Performance Evaluation of Noise Reduction during On-Line Monitoring of MV Switchgear for PD Measurements by Non-intrusive Sensors. Intl. J. of Electr. Powr. En. Sys., 64(1), pp. 596-607. 
[8] Hussain, G.A; Shafiq, M.; Lehtonen, M., Hashmi, M., 2015. Online Condition Monitoring of MV Switchgear by D-dot Sensor to Predict Arc-faults. IEEE Sensors Journal, 15(12), pp. 7262-7272.

[9] Hao, Z.; Blackburn, T.R; Phung, B.T; Liu, Z., Application of Signal Processing Techniques to On-line Partial Discharge Detection in Cables. Intl. Conference on Power System Tech., 21-24 Nov 2004.

[10] Maheswari, R.V.; Subburaj, P.; Vigneshwaran, B.; Iruthayarajan, M.W., 2014. Partial Discharge Signal Denoising using Adaptive Translation Invariant Wavelet Transform-Online Measurement. Journal of Electrical Engineering Technology, 9(2), pp. 695-706.

[11] Ma, X.; Zhou, C.; Kemp, I.J., 2002. Interpretation of Wavelet Analysis and Its Application in Partial Discharge Detection. IEEE Trans. on Dielec. and Electr. Insul., 9(3), pp. 446-457.

[12] Ghorat, M.; Gharehpetian, G. B.; Latifi H.; Hejazi, M.A; 2018. A New Partial Discharge Signal Denoising Algorithm Based on Adaptive Dual-Tree Complex Wavelet Transform. IEEE Trans. on Inst. and Meas., 67(10), pp. 2262-2272.

[13] Hussain, G.A.; Kumpulainen, L.; Klüss, J.V.; Lehtonen, M.; Kay, J.A.; 2013. The Smart Solution for the Prediction of Slowly Developing Electrical Faults in MV Switchgear Using Partial Discharge Measurements. IEEE Trans. on Power Delivery, 28(4), pp. 2309-2316.

[14] Jahangir, H.; Hajipour, E.; Vakilian, M.; Akbari, A.; Blackburn, T.; Phung, B.T., 2015. A Method to Capture and De-noise Partial Discharge Pulses Using Discrete Wavelet Transform and ANFIS. Intl. Trans. on Electr. Energy Sys., vol. 25. pp. 2696-2712.

[15] Liu, X., 1990. Partial Discharge Detection and Analysis in Low pressure environments”, Doctoral Dissertation, Ohio State University.

[16] Jian, L.; Tianyan, J.; Grzybowski, S.; Changkui, C., 2010. Scale Dependent Wavelet Selection for De-noising of Partial Discharge Detection. IEEE Trans. on Dielectr. Electr. Insul., 17(6), pp. 1705-1714.

[17] Ma, X.; Zhou, C.; Kemp, I.J., 2002. Automated Wavelet Selection and Thresholding for PD Detection. IEEE Electr. Insul. Magazine, 18(2), pp. 37-45. 
[18] Mitra, D.J; Shahjalal, M.; Kiber, M.A. 2014. Comparative Study on Thresholding Techniques of Discrete Wavelet Transform (DWT) to De-noise Corrupted ECG Signals. Intl. J. Engg. \& Computer Sci., 3(8), pp. 7605-7609.

[19] Hikita, M.; Okabe, S.; Murase, H.; Okubo, H., 2008. Cross-equipment evaluation of partial discharge measurement and diagnosis techniques in electric power apparatus for transmission and distribution. IEEE Trans. Dielec. \& Electr. Insul., 15(2), pp. 505-518. 


\section{Appendix A}

Highlighting the measurement principals of both HFCT and $D$-dot sensors, it is described that both are non-intrusive sensors. HFCT senses the PD signals using magnetic field, while $D$-dot sensors senses the PD signals using electric field. Once the signal is sensed, it comes across the electrical behavior of the respective sensors based on their respective geometrical parameters that determines electrical $(R L C)$ parameters.

\section{A. Working of HFCT}

In Fig. A1, $V_{c}(t)$ is the voltage developed at the coil (winding) of the HFCT, which serves as a source voltage for the coil's equivalent circuit. In the presence of terminating impedance, operation of the HFCT can be expressed as:

$$
V_{c}(t)=M_{c} \frac{d i_{P D}(t)}{d t}
$$

Where $i_{P D}(t)$ is the PD signal to be measured and $M_{c}$ is the mutual inductance between the PD source and coil winding. The output of the HFCT in terms of transfer function can be expressed as:

$$
V_{c}(s)=\frac{\frac{1}{L_{c} C}}{s^{2}+\frac{1}{L_{c} C}\left(\frac{L_{c}}{R_{t}}+R_{c} C\right) s+\frac{1}{L_{c} C}\left(\frac{R_{c}}{R_{t}}+1\right)} M_{c} s I_{P D}(s),
$$

Equation (A.2) gives a complete representation of the operation involved between $i_{P D}$ and $V_{c}$, where $s I_{D P}(s) \stackrel{L^{-1}}{\longrightarrow} \frac{d i_{P D}(t)}{d t}$ and $R_{c}, L_{c}, C_{c}$, and $R_{t}$ are coil's resistance, inductance, capacitance, and damping (terminating) resistance as shows in Fig. A1. 


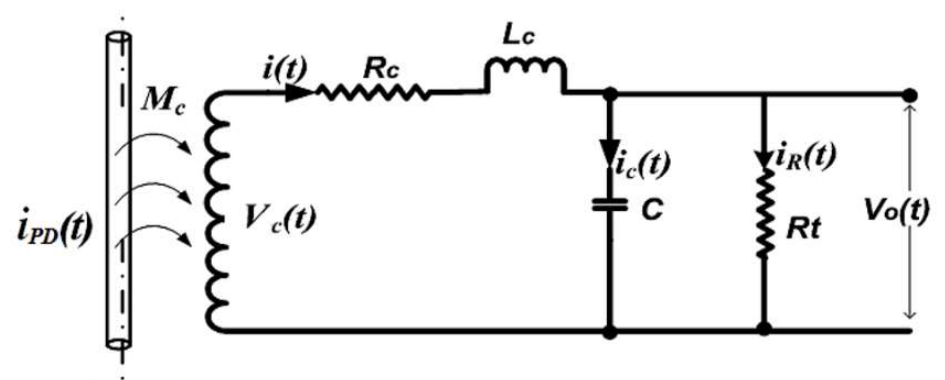

PD signals

Fig. A1. Electrical equivalent model of the HFCT

\section{A. Working of the D-dot Sensor}

The $D$-dot sensor is a capacitive sensor. The working principle of the sensor is demonstrated in Fig. A2 (a), and its electrical equivalent model is shown in Fig. A2 (b). The output current of the sensor can be represented by

$i(t)=p C \frac{d E(t)}{d t}$

where $C$ is the capacitance between the discharge source and the centre conductor of the sensor head and $p$ is the distance between them. The electric potential and the electric field are correlated as $V(t)=p \cdot E(t)$. The electrical model of $D$-dot sensor is the second order $R L C$ circuit as presented by the HFCT, therefore the transfer function of the $D$-dot sensor can be similar to the one given in equation A.2. Further details of the $D$-dot sensor can be found in [7]. 


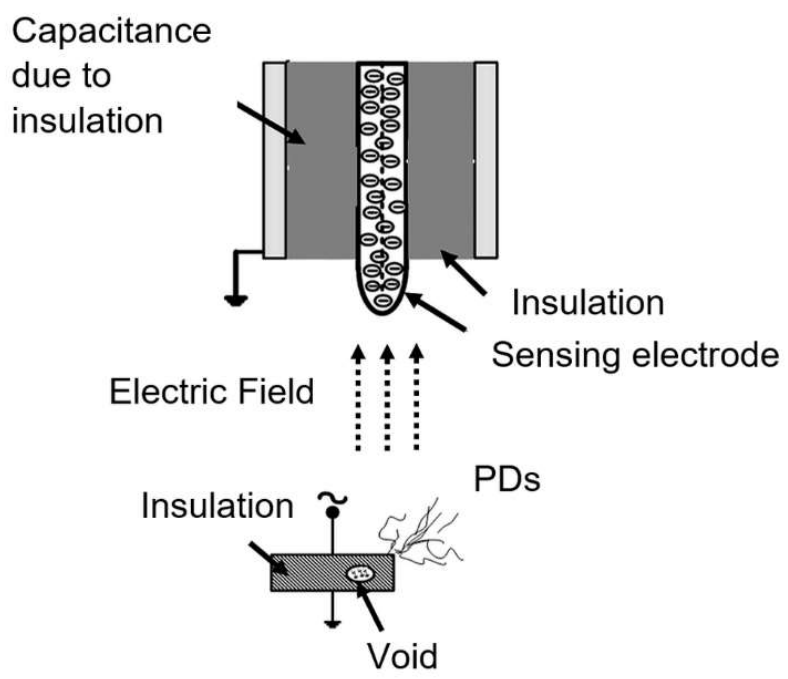

(a)

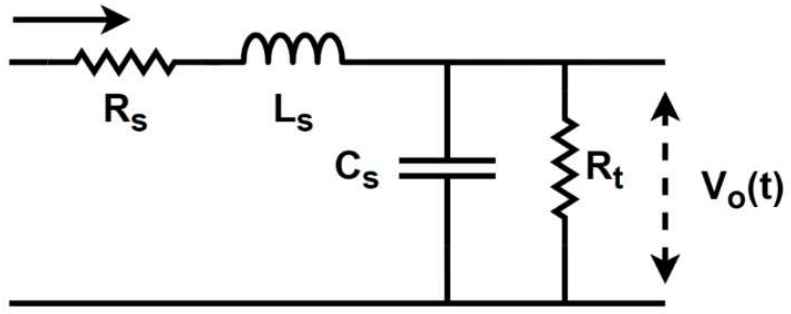

(b)

Fig. A2. D-dot Sensor, (a) Working principle, (b) Electrical equivalent model 


\section{List of Figure Captions:}

Fig. 1. PD Measurement setup

Fig. 2. Various wavelet functions

Fig. 3 (a) DWT based Multiresolution signal decomposition; (b) Reconstruction to obtain the denoised signal

Fig. 4. Defining number of decomposition level using ESD

Fig. 5 Correlation Coefficient for different wavelet functions

Fig. 6(a). PD pulse measured by HFCT sensor

Fig. 6(b). Wavelet function 'db3'

Fig. 7. Flow chart of self adaptive de-noising in online condition monitoring

Fig. 8. Performance indices against various frequency and amplitude limits

Fig. 9. PD Signal to be analyzed (a) the PD signal measured in laboratory (without noise), (b)

Simulated white noise, (c) PD signal mixed with noise

Fig. 10. De-noising based on EBWS

Fig. 11. De-noising based on CBWS

Fig. 12. FFT of signal S1

Fig. 13 Decomposition of signal S1 using db2 and 15 levels

Fig. 14. (a) PD signal captured by the sensor, (b) the PD signal with added noise, (c) De-noised signal, (d) Comparison between the measured PD signal and the de-noised signal (after removing the added noise)

Fig. 15. Performance indices for 200 signals de-noised by the adaptive de-noising technique, (a) correlation coefficient, (b) Mean square error, (c) Reduction in PD pulse amplitude, (d) Reduction in noise level, (e) Signal to noise ratio

Fig. A1. Electrical equivalent model of the HFCT

Fig. A2. D-dot Sensor, (a) Working principle, (b) Electrical equivalent model 